\title{
Ocorrência de intermitência na transição laminar-turbulenta em um escoamento de Couette plano
}

\author{
Occurrence of intermittency in a laminar-turbulent transition in a plane Couette \\ flow
}

\author{
Jean Jonathan Schuster ${ }^{1}$, Cristiano Henrique Schuster ${ }^{1}$, Eduardo Stüker ${ }^{1}$, Áttila Leães Rodrigues ${ }^{1}$, \\ Luiz Eduardo Medeiros ${ }^{1}$, Felipe Denardin Costa $^{1}$
}

Programa de Pós Graduação em Engenharia, Universidade Federal do Pampa, Campus Alegrete, Alegrete, RS, Brasil. jeanjschuster@gmail.com; cris.h.schuster@gmail.com; eduardostuker@gmail.com; attila.leaes@gmail.com; luizmedeiros@ unipampa.edu.br; fdenardin@unipampa.edu.br

\begin{abstract}
Resumo
A transição de regime de escoamento laminar-turbulenta é importante na maioria das áreas de aplicação de mecânica de fluidos. Na camada limite planetária (CLP), o escoamento é predominantemente turbulento. Todavia, logo após o ocaso, a incidência de radiação solar cessa e a superfície passa a perder calor através da emissão de ondas longas, dando origem a uma camada limite estavelmente estratificada (CLE), onde a turbulência pode ser suprimida praticamente em todas as suas escalas. Nestas condições a produção de turbulência é predominantemente mecânica, sendo que em noites com forte estratificação, a atividade turbulenta é reduzida em várias ordens de grandeza, podendo ressurgir abruptamente de forma imprevisível, dando orgiem a um fenômeno conhecido como intermitência global. A intermitência global é um fenômeno que ocorre na transição do escoamento na CLP, de forma similar a intermitência que ocorre na transição laminar-turbulenta. Desta forma, este trabalho visa o desenvolvimento de um experimento numérico para reproduzir a transição laminar-turbulenta em um escoamento de Couette estratificado termicamente, utilizando um modelo simulação de grandes turbilhões. As simulações mostram que para uma determinada faixa de parâmetros, durante a transição-laminar turbulenta, a turbulência surgiu de forma intermitente no escoamente.
\end{abstract}

Palavras-chave: Transição laminar-turbulenta, intermitência, camada limite estável, simulação de grandes turbilhões.

\begin{abstract}
The transition from laminar-turbulent flow regime is important in most of the fluid mechanics application areas. In the planetary boundary layer $(P B L)$, the flow is predominantly turbulent. However, shortly after sunset, the incidence of solar radiation ceases and the surface begins to lose heat through the emission of long-wave, yielding in a thermical stratified stable boundary layer (SBL), where turbulence can be suppressed in almost all scales. Under these conditions the production of turbulence is predominantly mechanical, and at nights with strong stratification, the turbulent activity is reduced by several orders of magnitude and can rise abruptly in unpredictable ways, giving origin to a phenomenon known as global intermittency. The globla intermittency is a phenomenon that occurs in the transition flow in the PBL, similarly to intermittency which occurs in the laminar-turbulent transition. Thus, this work aims to develop a numerical experiment to reproduce the laminar-turbulent transition in a thermally stratified Couette flow, using a large eddy simulation model. The simulations show that for a certain range of parameters during the transition laminar-turbulent, turbulence appeared intermittently in the flow.
\end{abstract}

Keywords: Laminar-turbulent transition, intermittency, stable boundary layer, large eddy simulation. 


\section{Introdução}

Logo após o pôr-do-Sol a incidência de radiação de onda curta sobre a superfície terrestre cessa, e essa passa por um processo de resfriamento radiativo devido à emissão de radiação de onda longa. Como consequência deste processo, as camadas de ar adjacentes à superfície também são resfriadas, dando origem a uma camada limite estratificada conhecida como camada limite estável (CLE) (Stull, 1988; Arya, 2001). Em tais condições a produção de turbulência é exclusivamente mecânica, através do cisalhamento do vento, enquanto que o empuxo, que durante o dia atua na geração de turbulência, neste caso é um sumidouro de turbulência devido a estratificação da CLE. Em noites de céu claro e com pouco vento a perda radiativa da superfície é intensa ocasionando uma estratificação térmica capaz de suprimir em muitas escalas de grandeza a atividade turbulenta (Mahrt e Vickers, 2006). Afetando diretamente os processos difusivos que ocorrem na camada limite atmosférica (CLA).

Tais condições representam um desafio para modelos de previsão do tempo, pois a estimativa dos fluxos turbulentos torna-se muito difícil, uma vez que a estratificação térmica atua na diminuição da intensidade da turbulência, muitas vezes sendo esta suprimida completamente, causando um comportamento intermitente do escoamento. Este fenômeno é chamado de intermitência global, e é ocasionado pela transição do regime de escoamento.

Assim como acontece na atmosfera, a transição de escoamento laminar-turbulenta ocorre de forma intermitente. Assim, a presente proposta visa o desenvolvimento de um experimento numérico para o estudo da transição utilizando um software livre de fluidodinâmica computacional, para reproduzir um escoamento de Couette estavelmente estratificado.

\section{Metodologia}

O experimento numérico consiste em um escoamento de Couette de um fluido compressível, onde o domínio onde ocorreu a discretização das equações é constituído em duas placas planas paralelas, distanciadas a $2 \mathrm{~m}$ uma da outra, onde a placa inferior é imóvel enquanto a placa superior tem velocidade constante. A largura do domínio é de $2 \mathrm{~m}$, enquanto o comprimento é de 4 metros. O domínio é dividido por uma malha de (40 X $50 \times 30$ ). As fronteiras de entrada e saída do escoamento são ortogonais a direção do escoamento e as fronteiras laterais são paralelas a direção do escoamento.

As seguintes condições foram adotadas para as fronteiras:

- as fronteiras inferior e superior representam contornos sólidos, sendo a velocidade relativa entre as fronteiras e o fluido nula;

- durante os primeiros $400 \mathrm{~s}$ de simulação a fronteira superior e inferior estavam a $300 \mathrm{~K}$, após estes 400 $\mathrm{s}$ a temperatura da placa inferior foi diminuída em $0,8 \mathrm{~K}$;

- as demais fronteiras são do tipo cíclicas, onde uma partícula deixando o domínio volta a entrar no mesmo pela fronteira oposta.

O modelo de turbulência utilizado é um modelo de simulação de grandes turbilhões (LES) (do inglês Large Eddy Sumulation), de uma equação de turbulência, tal qual o modelo proposto por Smagorinsky (Rieth et al., 2014). Porém, o modelo utilizado neste trabalho resolve uma equação de transporte adicional para a energia cinética turbulenta (ECT) para as escalas de sub grade (ESG). A descrição completa desse modelo pode ser obtida na biblioteca oneEqEddy.C do software OpenFOAM $\AA$, que será a plataforma utilizada neste trabalho, juntamente com o solver, presente nesta mesma plataforma, para escoamento turbulento, transiente, com transferência de calor para fluidos compressíveis buoyantPimpleFoam.

As propriedades utilizadas para o fluido da simulação foram equivalentes a do ar para uma temperatura de $300 \mathrm{~K}$.

Foram realizadas 6 simulações distintas, onde a única diferença entre as simulações foi a velocidade da placa superior. Estas velocidades foram $\bar{U}=0,2 \mathrm{~m} \mathrm{~s}^{-1}$, $\bar{U}=0,4 \mathrm{~m} \mathrm{~s}^{-1}, \bar{U}=0,6 \mathrm{~m} \mathrm{~s}^{-1}, \bar{U}=0,8 \mathrm{~m} \mathrm{~s}^{-1}, \bar{U}=1$ $\mathrm{m} \mathrm{s}^{-1}$ e $\bar{U}=1,2 \mathrm{~m} \mathrm{~s}^{-1}$. Para os subcapítulos 3.1 e 3.2 , serão apresentados apenas o caso em que a velocidade da fronteira superior foi $\bar{U}=0,6 \mathrm{~m} \mathrm{~s}^{-1}$ (Caso 1), $\bar{U}=0,8$ $\mathrm{m} \mathrm{s}^{-1}$ (Caso 2) e $\bar{U}=1,2 \mathrm{~m} \mathrm{~s}^{-1}$ (Caso 3), devido ao fato da simulação com velocidade da fronteira superior a $\bar{U}=0,8 \mathrm{~m} \mathrm{~s}^{-1}$, ou Caso 2, apresentar comportamento intermitente, por isso, para as séries temporais e para os perfis médios optou-se por mostrar os dados da primeira simulação anterior ao Caso 2 , em que houve a laminarização do escoamento (Caso 1), e pela simulação com maior turbulência (Caso 3).

Inicialmente as simulações ocorreram durante 400 $\mathrm{s}$ afim de garantir que o escoamento fosse turbulento, para que após estes 400 segundos iniciais, introduzir-se um gradiente de temperatura no domínio diminuindo a temperatura da fronteira inferior de 300 para $299,2 \mathrm{~K}$. A partir deste momento as simulações ocorreram durante mais 10800 segundos.

Os pontos de amostragem estão localizados no centro do domínio, sendo estes 19 pontos distanciados a $0,1 \mathrm{~m}$ um do outro, estando dispostos verticalmente um sobre o outro. A taxa de amostragem das simulações é de 4 $\mathrm{Hz}$. 


\section{Resultados}

A partir das simulações obteve-se o campo de velocidade para as três componentes do campo de velocidade do escoamento, além do campo de temperatura. De posse desses dados foram criadas séries temporais para as componentes horizontais do escoamento e para a temperatura, onde cada ponto nas análises representa um agrupamento de 4 observações. Ademais, também serão apresentados os perfis médios de velocidade, temperatura e ECT.

\subsection{Séries temporais}

O primeiro caso apresetado (Caso 1), é a simulação em que a velocidade da fronteira superior foi de $\bar{U}=0,6$ $\mathrm{m} \mathrm{s}^{-1}$. Observa-se que após a introdução do gradiente de temperatura entre as fronteiras superior e inferior, o período transiente estendeu-se até aproximadamente 3000 s, e após esse período transiente as flutuações das componentes de velocidade horizontal sessam. Ademais, observou-se grande diminuição da magnitude da componente de velocidade na direção do escoamento, enquanto a componente perpendicular ao escoamento tornou-se praticamente nula, como mostra a Figura 1.

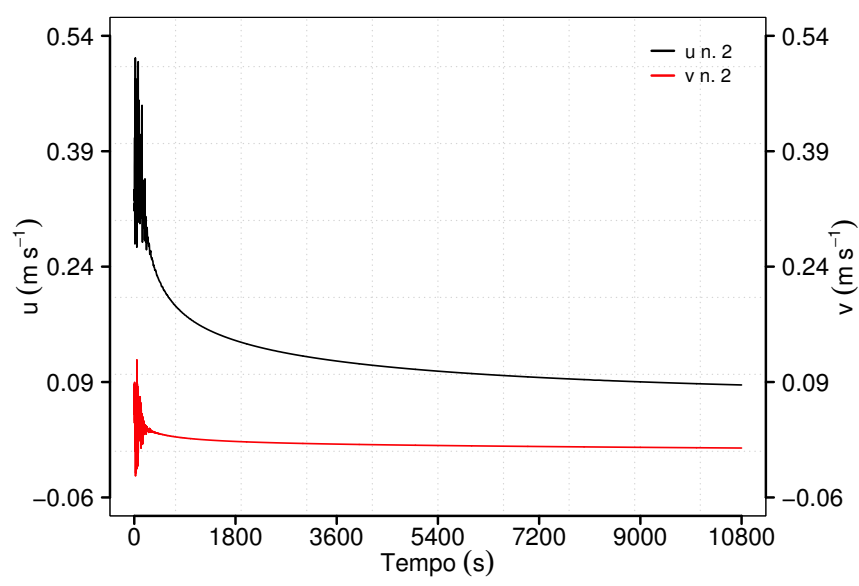

Figura 1: Série temporal das componentes horizontais de velocidade no nível 2 - Caso 1.

Já na série temporal de temperatura mostrada na Figura 2, fica evidente que após a aplicação do gradiente térmico na simulação, o escoamento que antes era isotérmico, tornou-se estavelmente estratificado $(\partial T / \partial Z>0)$. Após o período transiente a temperatura permaneceu constante em todos os níveis indicando que o escoamento tornou-se laminar completamente desenvolvido térmica e hidrodinamicamente (Martinez, 2006).

O segundo caso apresentado (aqui referenciado como Caso 3, em virtude do valor da velocidade da placa superior) em que a velocidade da fronteira superior foi

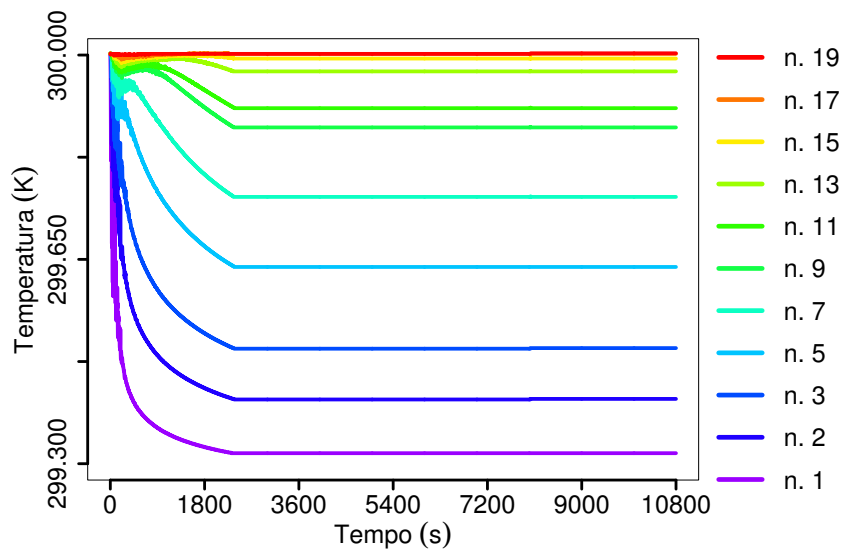

Figura 2: Evolução temporal da temperatura em todos os níveis de análise - Caso 1.

de $\bar{U}=1,2 \mathrm{~m} \mathrm{~s}^{-1}$, mostrado na Figura 3, a turbulência manteve-se mesmo após a inserção do gradiente de temperatura. Além disso, após a aplicação do gradiente de temperatura, houve uma pequena diminuição nas escalas de turbulência, onde as flutuações de velocidade estenderam-se durante todo o período simulado.

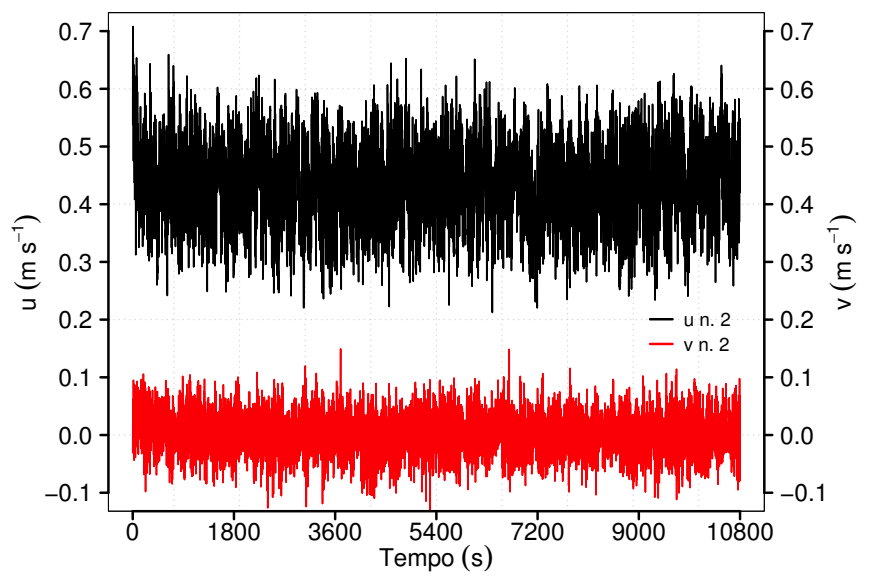

Figura 3: Série temporal das componentes horizontais de velocidade no nível 2 - Caso 3.

Ainda para o Caso 3, a série temporal de temperatura mostrada pela Figura 4, evidencia grandes processos de difusão de calor oriundos de intensas flutuações de velocidade característico de escoamentos turbulentos (White, 2015).

O terceiro caso apresentado (referênciado como Caso 2 devido ao valor de velocidade nesta simulação ser intermediário aos dois casos anteriores), em que a velocidade da fronteira superior foi de $\bar{U}=0,8 \mathrm{~m} \mathrm{~s}^{-1}$, mostrou-se intermitente, conforme observado pela Figura 5. Após a aplicação do gradiente de de temperatura entre as fronteiras ouve uma drástica diminuição das escalas de turbulência, apresentando pequenas explosões de turbulência (bursts) em intervalos irregulares. Em 


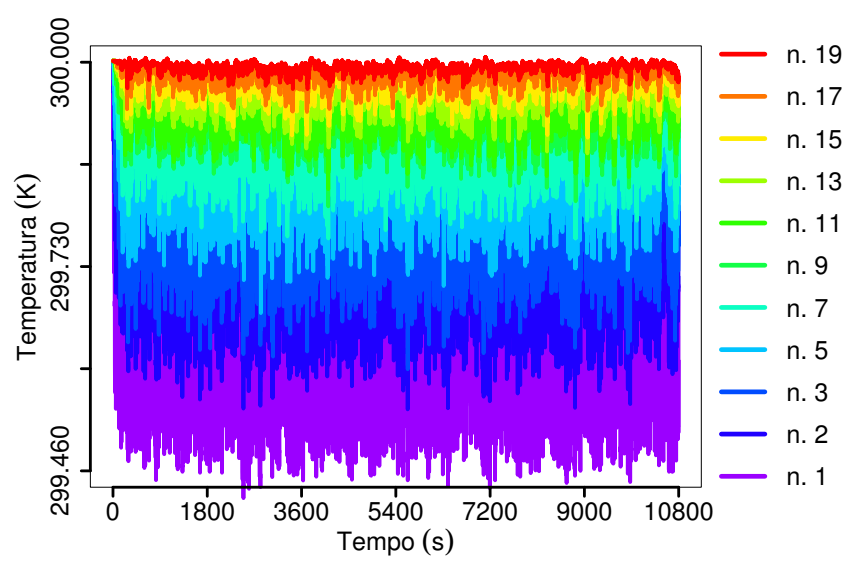

Figura 4: Evolução temporal da temperatura em todos os níveis de análise - Caso 3.

torno de $8000 \mathrm{~s}$ de simulação ocorreu o primeiro evento em que as escalas de turbulência tiveram um aumento abrupto de amplitude, estendendo-se até aproximadamente $9500 \mathrm{~s}$, caracterizando a mudança de regime de escoamento para totalmente turbulento durante este período. Após este primeiro evento de transição completa de regime, foi possível observar mais seis eventos até o final do período simulado, em $36000 \mathrm{~s}$.

Fenômenos semelhantes são observados na atmosfera, conforme evidenciado por Sun et al. (2012) em um trabalho realizado analisando os dado obtidos pelo experimento CASES-99.

As perturbações de velocidade causadas por estes eventos turbulentos são observadas em ambas as componentes horizontais de velocidade. A ocorrência destes bursts intermitentes já foi observada em diversos experimentos reproduzindo um regime transitório, um destes apresentado por Schlichting et al. (2000).

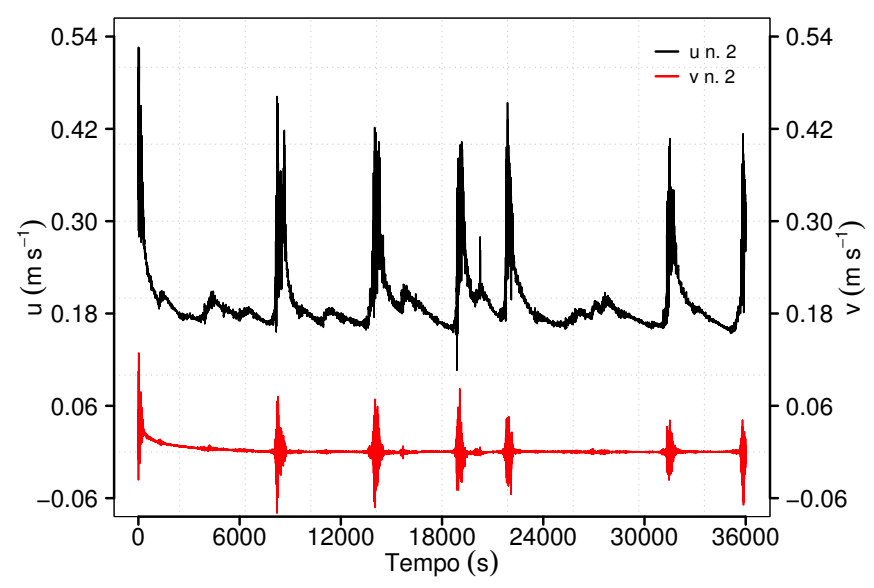

Figura 5: Série temporal de das componentes horizontais de velocidade no nível 2 - Caso 2.

Estas perturbações no regime de escoamento, ocorridas no Caso 2, também podem ser vistas na série tem- poral de temperatura, Figura 6, onde observa-se maior fluxo de calor da fronteira superior para a inferior nos eventos turbulentos. Isto deve-se aos processos de difusão de calor e momento desencadeados durantes os eventos de maior turbulência, onde observa-se o acoplamento energético entre os diferentes níveis.

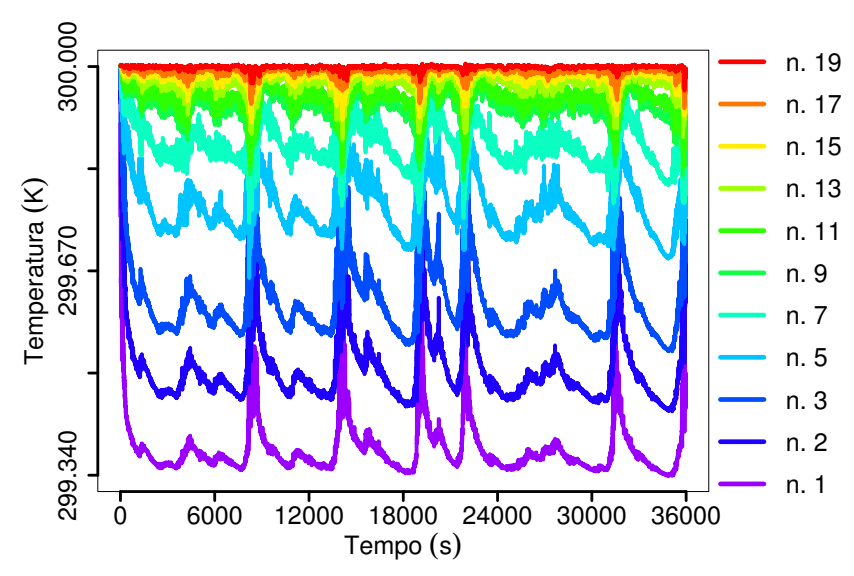

Figura 6: Evolução temporal da temperatura em todos os níveis de análise - Caso 2.

\subsection{Análise dos perfis verticais médios}

Os perfis médios de velocidade, temperatura e ECT foram obtidos através da média dos valores do campo para a última hora de simulação.

É possível observar, através da Figura 7, que o perfil de velocidade para o Caso 1 é praticamente linear, indicando escoamento laminar. Já o Caso 3 demonstra um perfil turbulento para a metade inferior e superior do domínio, indo ao encontro dos resultados obtidos por Lagha e Manneville (2007) em um modelo proposto pelos autores, e em simulação por direct numerical simulation (DNS) por Pirro e Quadrio (2008). O Caso 2, por sua vez, mostra um perfil intermediário entre o laminar e o turbulento, característico de escoamentos transitórios, onde observa-se maior turbulência na metade superior do domínio, já no metade inferior do domínio a curvatura do perfil é menor, indicando uma mescla entre regimes turbulentos de escalas diferentes, ou alternância entre regimes laminares e turbulentos, conforme observado na série temporal de velocidade mostrada na Figura 5.

Quanto ao regime de escoamento, os perfis de temperatura mostrados pela Figura 8 , onde $\Theta$ é a razão entre a temperatura no nível e a temperatura da fronteira superior, indicam um comportamento mais linear para o Caso 1, enquanto o Caso 3 apresenta um perfil parabólico, já o Caso 2 apresenta valores intermediários entre os casos citados anteriormente. Esse comportamento é esperado, uma vez que quanto maior a turbulência no escoamento, maior os processos de transporte, sendo 


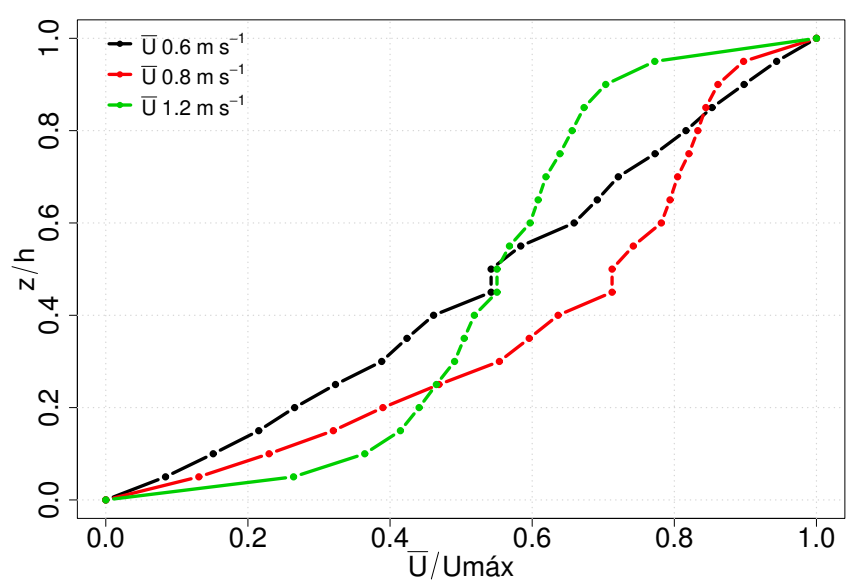

Figura 7: Perfis adimensionais de velocidade para o Caso 1 (preto), Caso 2 (vermelho) e Caso 3 (verde).

os efeitos da superfície da fronteira sentidos com maior intensidade no centro do domínio (Bergman et al., 2011).

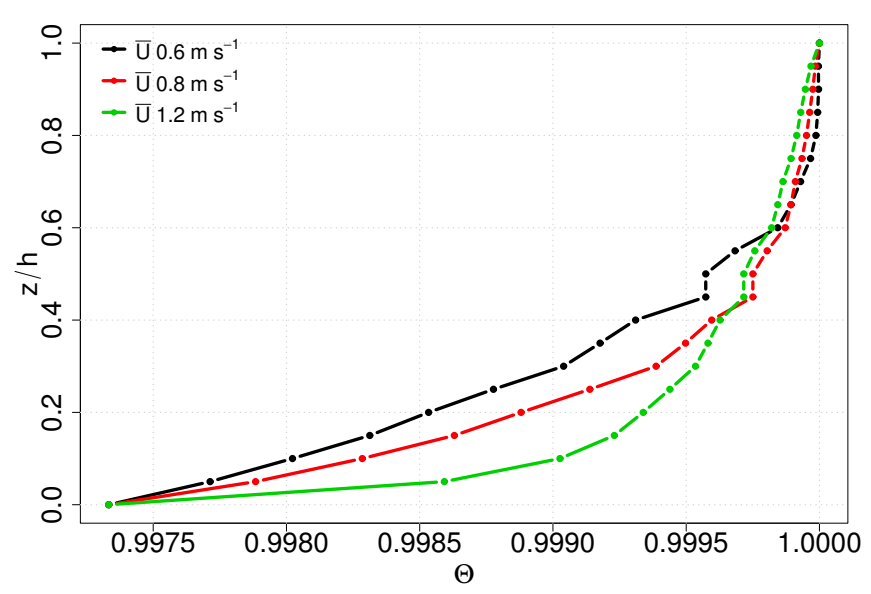

Figura 8: Perfis adimensionais de temperatura para o Caso 1 (preto), Caso 2 (vermelho) e Caso 3 (verde).

A partir do campo de velocidade criou-se os perfis médios de ECT (k). A ECT é obtida através da soma dos quadrados da flutuação das componentes de velocidade divididas por dois, ou seja, se não houver flutuações de velocidade em relação a velocidade média, não haverá ECT (Stull, 1988).

Com a ECT calculada, criou-se os perfis médios para os três casos, conforme visto na Figura 9. Como o esperado o Caso 3 mostrou maior turbulência, sendo esta com maior intensidade perto dos contornos sólidos. Já o Caso 2 mostrou uma maior turbulência na metade inferior do domínio, apresentando picos de turbulência próximo aos contornos sólidos. O Caso 1 apresentou valores de ECT praticamente nulos, indicando escoamento laminar. Estes resultados são condizentes com os resultados obtidos por DNS para um escoamento de Couette, onde Pirro e Quadrio (2008) analisaram a variância das flutuações de velocidade no domínio.

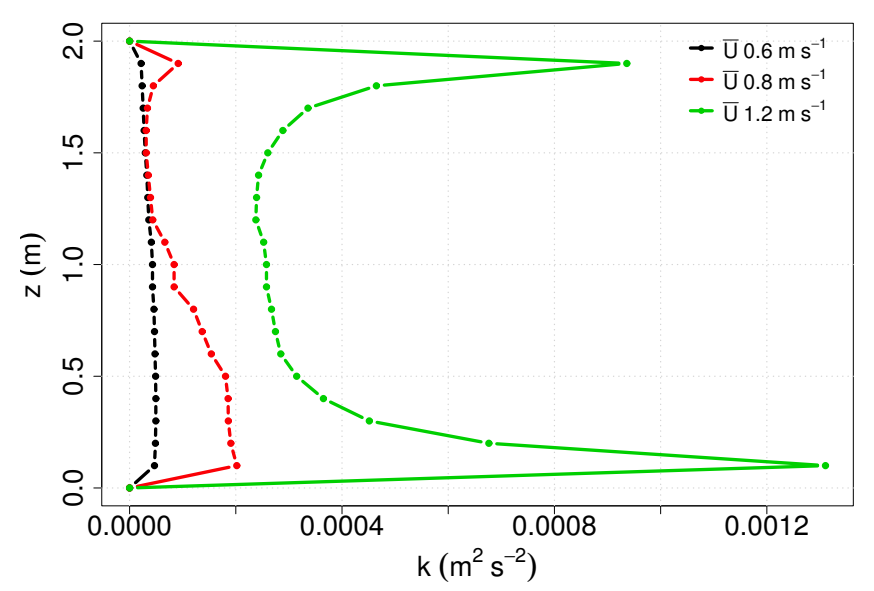

Figura 9: Perfis de ECT para o Caso 1 (preto), Caso 2 (vermelho) e Caso 3 (verde).

\subsection{Análise da transição}

Para uma primeira análise criou-se um gráfico de temperatura média para o nível 2 para cada simulação, conforme visto na Figura 10. Este gráfico mostra claramente o aumento da temperatura no nível quando o escoamento passa do regime laminar para o regime turbulento, isso ocorre pelo fato de a turbulência gerar grande difusão de momento e temperatura, levando à mistura do escoamento dentro do domínio. A temperatura tem um aumento abrupto a partir do caso com velocidade da placa superior de $\bar{U}=0,6 \mathrm{~m} \mathrm{~s}^{-1}$, indicando que os casos com velocidade maiores não estão em regime laminar. Este resultado pode ser interpretado da seguinte maneira: no regime laminar todos os níveis estão energeticamente desacoplados, o que faz com que a temperatura dos níveis inferiores seja próxima da temperatura da placa correspondente. Quando o regime é turbulento todos os níveis estão energeticamente acoplados pela turbulência, o que faz com que os efeitos de fronteira sejam sentidos em regiões mais distantes das respectivas placas. Assim, regiões mais próximas a placa quente se tornarão mais frias e o contrário acontece próximo a placa fria.

Olhando somente para o limite inferior do domínio a Figura 10 pode ser interpretada como a representação, ainda que para um caso idealizado, do acoplamento atmosférico (Acevedo et al., 2012). Onde um comportamento similar é observado na dependência da temperatura com a velocidade do vento.

Com o intuito de verificar a transição de regime optou-se pelo cálculo da velocidade de fricção $\left(u_{*}\right)$, que é a raiz quadrada do fluxo turbulento de momentum por unidade de massa, portanto quando $u_{*}$ for zero não existem flutuações de velocidade no escoamento, o 


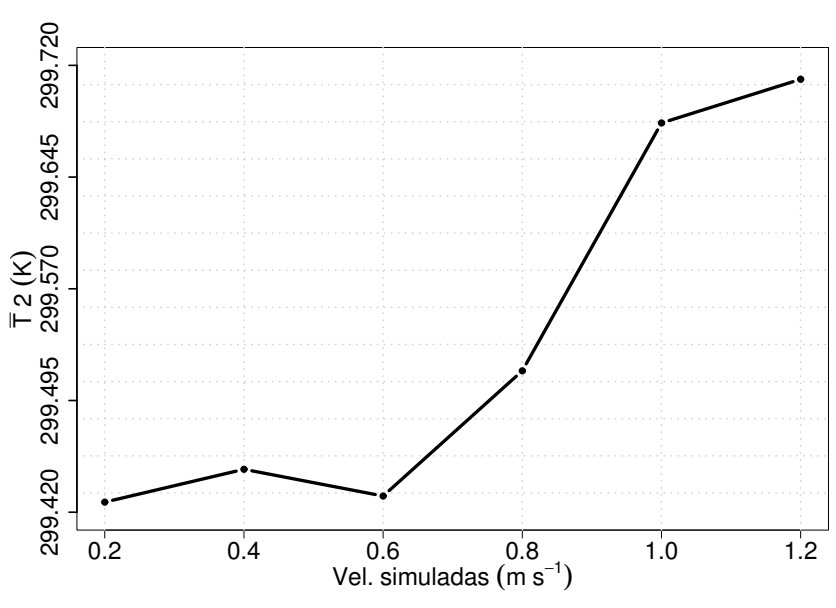

Figura 10: Temperatura média no nível 2 para cada uma das simulações.

que caracteriza o escoamento em regime laminar (Stull, 1988). A Figura 11 mostra a dependência de $u_{*}$ com a velocidade média do escoamento nos níveis um ao nove. Uma transição de regime similar ocorre na natureza durante a transição do regime de escoamento muito estável para o regime de escoamento pouco estável na CLE (Sun et al., 2012).

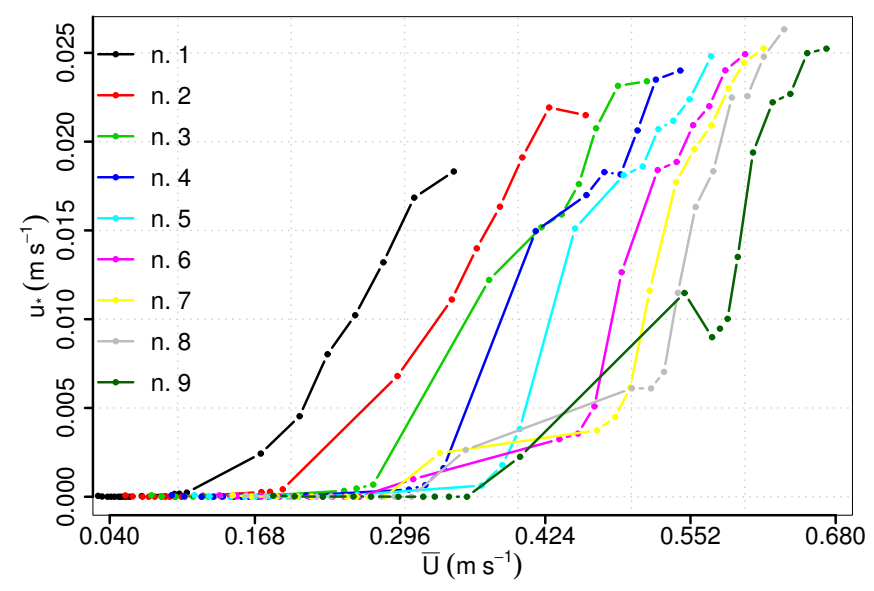

Figura 11: Vel. de fricção pela magnitude das componentes horizontais do escoamento (cada ponto consiste em um agrupamento de 200 observações).

O parâmetro utilizado para quantificar a transição do regime de escoamento foi o número Richardson gradiente. O número de Reynolds não foi utilizado para essa análise pois mesmo o escoamento laminar possui o Re muito alto (por exemplo, para o caso onde a velocidade da placa superior é igual a $\bar{U}=0,2 \mathrm{~m} \mathrm{~s}^{-1} \mathrm{o}$ Re é da ordem 40000). Desta forma a transição entre os regimes de escoamento foi analisado através da dependência de uma variável que quantifica a intensidade turbulenta $\left(u_{*}\right)$ e um parâmetro que representa a influência da estratificação estável no escoamento, neste caso o número de Richardson gradiente $(R i)$. O Ri relaciona a capacidade estabilizadora dos gradientes verticais de densidade, com a capacidade desestabilizadora do cisalhamento do vento, conforme observado pela Figura 12 (Arya, 2001). Cada ponto observado na Figura 12 corresponde a um agrupamento de 10 segundos, onde foram usados os dados de todas a seis simulações. Nota-se a ocorrência de mais de um regime de escoamento, e a transição entre os regimes de escoamento ocorre para $R i$ em torno de 0,2 como prevê a teoria (Nieuwstadt, 1984).

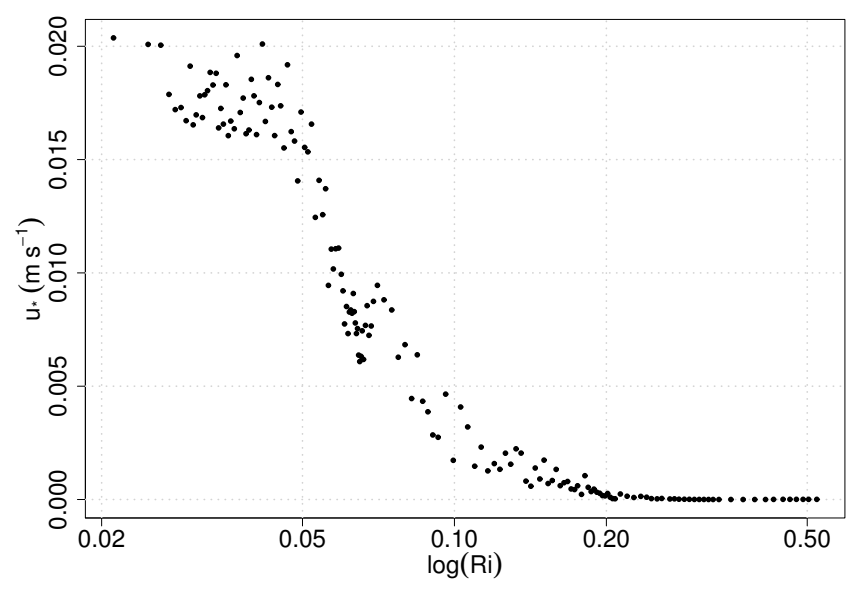

Figura 12: Vel. de fricção por log. de Richardson gradiente para todas as simulações (cada ponto representa um agrupamento de 40 observações).

\section{Conclusões}

Como principais resultados obtidos observou-se que quando o regime de escoamento é turbulento todos os níveis estão energeticamente acoplados pela turbulência, o que faz com que os efeitos de fronteira sejam sentidos em regiões mais distantes das fronteiras, enquanto no regime laminar todos os níveis estão energeticamente desacoplados, o que cria uma estratificação térmica. Observou-se também a mudança do regime laminar para o turbulento com o aumento da velocidade média do escoamento, além da evidencia de vários regimes de escoamento nos casos simulados, bem como a transição de regime, quando $R i$ atinge o valor crítico igual a 0,2 .

Quanto a proposta do desenvolvimento de um experimento numérico para o estudo da transição de regime de escoamento laminar-turbulenta, considera-se o objetivo como atingido, pois foi possível observar aspectos característicos da transição de escoamento, mesmo utilizando um dos modelo de turbulência LES mais simples disponíveis.

Um desafio a ser abordado no futuro é a caracterização mais completa da transição laminar-turbulenta, 
utilizando um modelo mais completo fisicamente. Além disso, pretende-se avaliar aspectos e condições que favoreçam a transição e quais são os principais fatores que contribuem para o surgimento dos eventos intermitentes de turbulência, característicos desse regime de escoamento.

\section{Agradecimentos}

Os autores agradecem às agências CAPES (Coordenação de Pessoal de Nível Superior) e CNPq (Conselho Nacional de Desenvolvimento Científico e Tecnológico) e à Universidade Federal do Pampa pelo suporte financeiro. O cluster computacional utilizado nas simulações foi adquirido com recursos do Edital 02/2014 - PqG (Edital Pesquisador Gaúcho) da Fundação de Amparo a Pesquisa do Estado do Rio Grande do Sul (FAPERGS).

\section{Referências}

Acevedo, O. C., Costa, F. D., Degrazia, G. A. (2012). The coupling state of an idealized stable boundary layer. Boundary-layer meteorology, 145(1), 211-228.

Arya, P. S. (2001). Introduction to micrometeorology, vol 79. Academic press.

Bergman, T. L., Incropera, F. P., Lavine, A. S. (2011). Fundamentals of heat and mass transfer. John Wiley \& Sons.

Lagha, M., Manneville, P. (2007). Modeling of plane couette flow. i. large scale flow around turbulent spots. Physics of Fluids (1994-present), 19(9), 094,105.

Mahrt, L., Vickers, D. (2006). Extremely weak mixing in stable conditions. Boundary-layer meteorology, 119(1), 19-39.

Martinez, D. M. V. (2006). Transição à turbulência na camada de mistura estavelmente estratificada utilizando simulação numérica direta e simulação de grandes escalas. .

Nieuwstadt, F. T. (1984). The turbulent structure of the stable, nocturnal boundary layer. Journal of the Atmospheric Sciences, 41(14), 2202-2216.

Pirro, D., Quadrio, M. (2008). Direct numerical simulation of turbulent taylor-couette flow. European Journal of Mechanics-B/Fluids, 27(5), 552-566.

Rieth, M., Proch, F., Stein, O., Pettit, M., Kempf, A. (2014). Comparison of the sigma and smagorinsky les models for grid generated turbulence and a channel flow. Computers \& Fluids, 99, 172-181.
Schlichting, H., Gersten, K., Gersten, K. (2000). Boundarylayer theory. Springer Science \& Business Media.

Stull, R. B. (1988). An introduction to boundary layer meteorology, vol 13. Springer Science \& Business Media.

Sun, J., Mahrt, L., Banta, R. M., Pichugina, Y. L. (2012). Turbulence regimes and turbulence intermittency in the stable boundary layer during cases-99. Journal of the Atmospheric Sciences, 69(1), 338-351.

White, F. (2015). Fluid mechanics. McGraw-Hill Higher Education. 